L'article présenté dans ces colonnes résume les points essentiels soulevés par le nouveau rapport d'Helsana sur l'«Evolution des dépenses en matière de santé en 2016». II nous éclaire sur l'évolution des dépenses de l'assurance obligatoire de soins en se fondant sur les données de prestations d'Helsana.

\title{
Evolution des dépenses en matière de santé
}

\section{Mathias Früh}

Politique et économie, groupe Helsana

1 OECD Health at a Glance: Europe 2016: 115/117 (http://bit.ly/2kOgolO). 2 www.helsana.ch/fr/ groupe-helsana/portraitde-notre-entreprise/ sciences-de-la-santepublique/rapport-sur-les depenses

La Suisse dispose d'un système de santé d'excellente qualité, voire le meilleur du monde en matière d'accès aux soins. Mais c'est bien connu, cela a un prix: les coûts de la santé ont augmenté de 10 milliards de francs en six ans (de 2008 à 2014) pour atteindre 71 milliards. Que ce soit par tête ou par rapport au produit intérieur brut, la Suisse occupe les premières places en comparaison internationale ${ }^{1}$, mais d'où proviennent ces coûts?

Les assurances-maladie, en tant que principaux répondants des coûts, sont intéressées par l'efficience et l'économicité de notre système de santé. Les données

\section{Utilisation du salaire brut mensuel dans un ménage moyen}

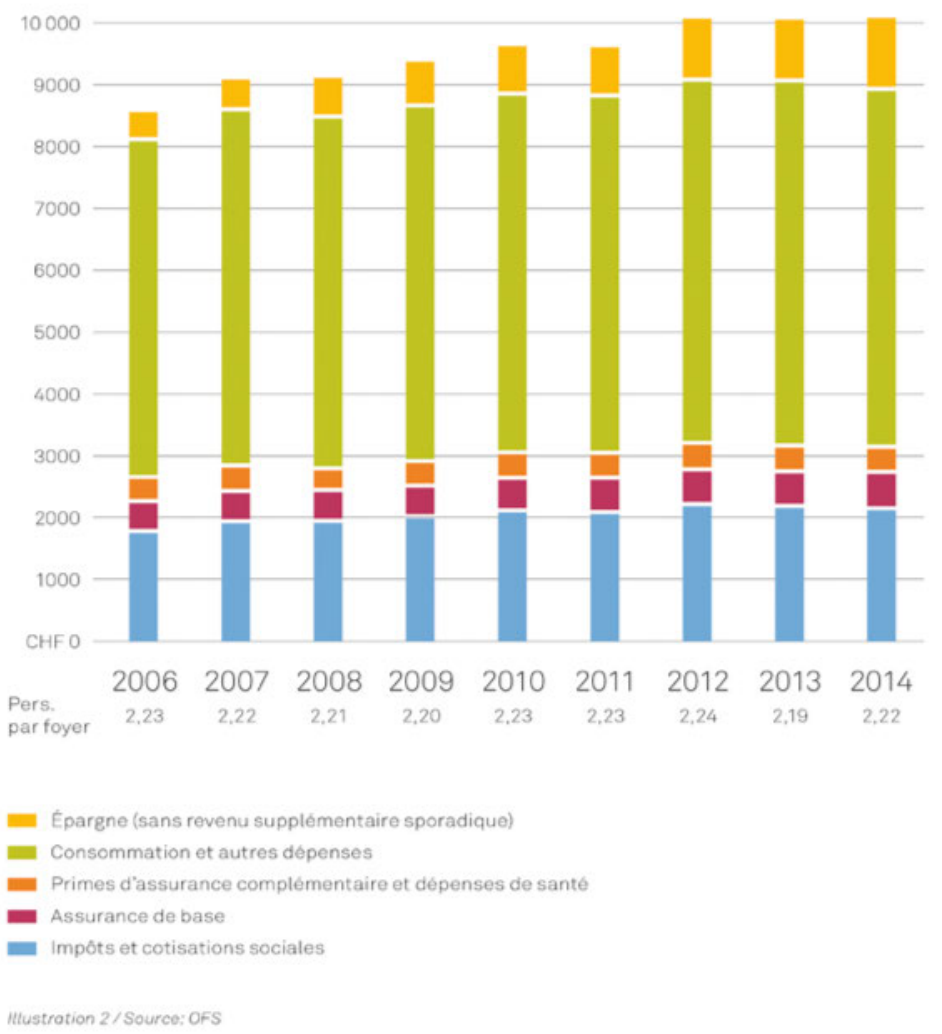

auxquelles nous avons accès permettent de porter un regard nuancé sur l'évolution des coûts et d'en nommer les raisons: elles mettent notamment en évidence le rôle essentiel de la démographie (la croissance démographique et le vieillissement de la population). La hausse des volumes et l'extension du catalogue des prestations sont d'autres causes de l'augmentation des coûts, tout comme de faux incitatifs systémiques qui peuvent intervenir, mais qui sont difficilement quantifiables. Les patients y contribuent également car ils veulent souvent, et si possible immédiatement, bénéficier du meilleur traitement.

Le rapport paru récemment «Evolution des dépenses en matière de santé $»^{2}$ apporte davantage de transparence dans le secteur des soins de base. Comme le rapport sur les médicaments, il se fonde sur les données de l'assurance obligatoire des soins du groupe Helsana et sur les statistiques accessibles au public. Les principales conclusions de ce rapport sont présentées ici pour discussion.

\section{L'économie suisse peut toujours mieux supporter l'augmentation des coûts de la santé}

Personne ne peut nier que la hausse annuelle des primes d'assurance-maladie grève le budget de beaucoup de ménages. En 2014, le poids de l'assurance de base sur le ménage moyen était de 585 francs par mois, soit 7020 francs par an. Au total, l'augmentation de la charge mensuelle de 2006 à 2014 s'élève à 100 francs environ. C'est beaucoup, mais finalement pas tant que ça si les 
chiffres sont remis dans le contexte: pendant la même période, la consommation mensuelle moyenne a augmenté de 325 francs, la charge fiscale a quadruplé et malgré tout, l'épargne mensuelle moyenne s'est accrue de 715 francs (cf. fig. 1).

Le grand problème ne réside pas dans la question de savoir si notre économie peut supporter les coûts mais dans la répartition des charges.

Cette considération basée sur des données agrégées constitue une moyenne; l'économie suisse peut toujours mieux supporter l'augmentation des coûts de la santé. Mais nous savons aussi que les moyennes ne signifient pas grand-chose pour le particulier. En effet, il existe toujours plus de ménages pour lesquels les primes d'assurance-maladie ont atteint un niveau qui n'est plus ou peu supportable. C'est aussi dans cette perspective qu'il faut considérer les discussions qui s'enflamment chaque année à ce sujet dans le débat public: le grand problème ne réside pas dans la question de savoir si notre économie peut supporter les coûts mais dans la répartition des charges.

Financement du système de santé selon le régime de financement

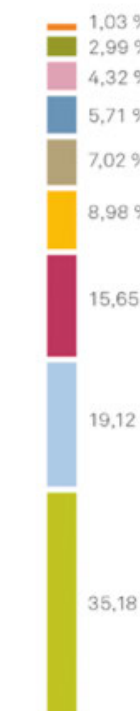

2008

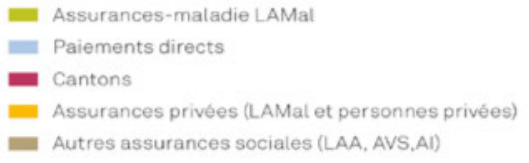

- Participations aux frais des assurés Etat-autres (Conféd. collect. etc.) - Personnes privées - autres
- Prestations sociales liées aux besoins

\section{Les prestations à la charge de l'assurance de base ne cessent d'augmenter...}

Si les coûts de la santé à la charge de la communauté ne cessent d'augmenter, c'est aussi lié au fait que le catalogue des prestations obligatoires ne cesse d'être élargi. La tendance veut que les formes de financement solidaires supplantent de plus en plus les formes de financement privées. La part de financement de l'assurance de base par rapport au total des coûts de la santé a augmenté entre 2012 et 2014 de près d'un milliard de francs (soit 36,6\% du total des coûts, cf. fig. 2).

\section{... et les patients bénéficient de soins plus fréquents et plus intensifs}

La majeure partie de la croissance des prestations d'assurance entre 2008 et 2015 est imputable à l'augmentation des coûts par patient. Alors que les prestations brutes ont augmenté de $33 \%$ au total, elles ont augmenté de $22 \%$ par patient. Cela signifie que les deux tiers de la croissance totale sont produits par la hausse des prestations par patient. Ces évolutions ne pouvant pas être seulement imputables aux changements démographiques, on peut émettre l'hypothèse qu'elles sont dues aux tarifs élevés, à une intensification des traitements, à des modifications du catalogue des prestations (p. ex. nouveaux médicaments plus chers) et/ ou à de nouvelles habitudes de facturation. Le tiers restant de l'augmentation des coûts de l'AOS provient de la hausse du nombre de patients, qui s'explique principalement par la croissance démographique.

\section{TARMED encourage une pratique non transparente en matière de facturation}

Venons-en au TARMED. Le tarif le plus important de Suisse pour le secteur ambulatoire génère un volume de plus de 11 milliards de francs. Plus de $40 \%$ des prestations TARMED (3,99 milliards de points tarifaires) sont issues du chapitre "Prestations de base». La majorité d'entre elles sont des consultations. Près de 15\% des prestations TARMED sont des examens d'imagerie médicale et $12 \%$ relèvent de la spécialité psychiatrie. Ces trois domaines "consultations», "imagerie médicale» et «psychiatrie» concentrent donc 7,4 milliards de francs (cf. tableau 1 ci-dessous)

Les prestations de base ont enregistré une hausse de $24 \%$ entre 2012 et 2015 . Le besoin a sans doute légèrement augmenté en raison de la croissance démographique mais les points tarifaires par patient ont simultanément progressé de $10 \%$. Cela suggère que, malgré l'intervention tarifaire du Conseil fédéral au profit des 


\section{Top 10 du nombre de points de taxation par chapitre (en millions de points de taxation)}

\begin{tabular}{|c|c|c|c|}
\hline Chapitre & 2012 & 2015 & Évolution depuis 2012 \\
\hline Total des points de taxation & 9584 & 11540 & $+20 \%$ \\
\hline 00 -Prestations de base & 3477 & 4943 & $+24 \%$ \\
\hline 39-Imagerie médicale & 1328 & 1753 & $+15 \%$ \\
\hline 02-Psychiatrie & 1328 & 1403 & $+19 \%$ \\
\hline 08-CEil & 535 & 683 & $+28 \%$ \\
\hline $\begin{array}{l}\text { 17-Diagnostic et traitement non chirurgical du } \\
\text { cœur et des vaisseaux }\end{array}$ & 321 & 375 & $+17 \%$ \\
\hline $\begin{array}{l}\text { 35-Salle d'opération, salle de réveil, } \\
\text { clinique de jour }\end{array}$ & 222 & 270 & $+22 \%$ \\
\hline 32-Radio-oncologie, radiothérapie & 243 & 270 & $+11 \%$ \\
\hline $\begin{array}{l}\text { 19-Diagnostic et traitement non chirurgical du } \\
\text { tractus gastro-intestinal }\end{array}$ & 219 & 264 & $+20 \%$ \\
\hline $\begin{array}{l}\text { 37-Pathologie clinique (autopsie, histologie, } \\
\text { cytologie) et médecine légale }\end{array}$ & 197 & 237 & $+20 \%$ \\
\hline 04-Peau, parties molles & 184 & 213 & $+16 \%$ \\
\hline
\end{tabular}

Tableau 1: Top 10 du nombre de points tarifaires par chapitre.

médecins de premier recours en 2014 (200 millions de francs), une grande partie de l'augmentation des coûts est due d'une part aux nouveaux comportements de facturation et d'autre part à des examens et des traitements plus approfondis.

\section{Nous nous engageons pour que les tarifs} trop élevés soient corrigés.

Tout le monde le sait, le TARMED doit être absolument révisé. Dans sa forme actuelle, il désavantage les médecins de premier recours au profit des spécialistes, il laisse trop de place à l'arbitraire dans la facturation et les imprécisions dans la tarification se traduisent par de faux incitatifs dans les soins.

Les adaptations urgentes que le Conseil fédéral veut ordonner sont nécessaires. Nous nous engageons pour que les tarifs trop élevés soient corrigés, la transparence accrue et les entretiens médicaux avec les patients revalorisés. Après l'intervention tarifaire, il s'agira de réviser rapidement et complètement la structure tarifaire ambulatoire et de l'adapter à la réalité actuelle.

\section{Il faut en finir avec la simple question des coûts et se concentrer sur la qualité et l'efficience}

Les données du secteur de la santé, tout comme celles sur lesquelles se base notre rapport sur les dépenses de santé, concernent principalement les prestations et les coûts. Aujourd'hui, les débats politiques portent essentiellement sur la hausse des coûts. Les acteurs essentiels - et notamment les décideurs en matière de politique de la santé - réagissent en premier lieu à l'augmentation des coûts, bouc émissaire de la problématique de répartition.

Il est bien évidemment primordial de maîtriser les coûts mais il est tout aussi nécessaire que le système de soins et la politique qui le façonne se projettent au-delà des dépenses et s'orientent davantage vers le bénéfice pour les patients et la qualité des traitements. Les mesures doivent être axées sur le point de vue de la qualité et de l'efficience. Il faut arriver à voir clairement la finalité, c'est-à-dire le résultat du traitement. C'est le seul moyen de surpasser une discussion purement sur les dépenses, car le secteur de la santé représente bien plus qu'un seul facteur de coûts. 